\title{
EFFICIENT RECALIBRATION VIA DYNAMIC MATRIX COMPLETION
}

\author{
Sofia Nikitaki ${ }^{1,2}$, Grigorios Tsagkatakis ${ }^{1}$ and Panagiotis Tsakalides ${ }^{1,2}$ \\ ${ }^{1}$ Institute of Computer Science, Foundation for Research and Technology - Hellas (FORTH-ICS) \\ 2 Department of Computer Science, University of Crete, \\ Heraklion, Crete, Greece \\ email:\{nikitaki,greg, tsakalid\}@ics.forth.gr
}

\begin{abstract}
Fingerprint-based localization techniques have witnessed significant progress as they provide highly accurate location estimation with minimal hardware interventions. However, the required calibration phase is time and labour consuming. In this work, we propose a reduced effort recalibration technique for fingerprint-based indoor positioning systems. Particularly, we reduce the number of received fingerprints by performing spatial sub-sampling. The recovery of the full map from partial measurements is formulated as an instance of a Dynamic Matrix Completion problem where we exploit the spatio-temporal correlations among the fingerprints. Analytical studies and simulations are provided to evaluate the performance of the proposed technique in terms of reconstruction and location error.
\end{abstract}

Index Terms - matrix completion, fingerprint-based indoor localization, recalibration, received signal strength measurements, spatio-temporal correlations.

\section{INTRODUCTION}

Location and mobility management are major functions for providing seamless and ubiquitous environments. Selforganizing ad-hoc networks, health care monitoring, personal tracking and efficient routing schemes are some of the potential applications [1].

The last decade, wireless technologies have entered the realms of indoor location based services. Particularly, IEEE 802.11, the dominant local wireless networking standard, is omnipresent and thus appealing to be used for localization purposes. The majority of indoor localization schemes exploit the power of the received signals, the so-called Received Signal Strength (RSS), in wireless local area networks (WLAN) as it provides a reliable and straightforward source of data. RSS-based localization systems can be classified into two

This work was funded by the CS-ORION (PIAP-GA-2009-251605) and HYDROBIONETS (ICT-GA-2011-287613) grants within the 7th Framework Program of the European Community. categories, namely fingerprint-based and distance-prediction based [1].

Distance-prediction based systems estimate the position of the mobile user by measuring its distances from at least three anchor nodes such as wireless Access Points (APs), wireless mesh routers, or sensor nodes [2]. These systems utilize a known RF propagation model in order to translate the signal information into an Euclidean distance. However, the power of the signal and thus the RSS measurements are affected from various multipath phenomena, low probability of the desirable line-of-sight path, and specific parameters (floor layout, moving objects), which make formulating a reliable radio propagation model a challenging task.

On the other hand, fingerprint-based localization systems create signature maps in order to represent the physical space by capturing the variations of the dynamic nature of indoor radio propagation $[3,4,5]$. These systems consist of two distinct phases: the calibration/training and the location estimation/runtime phase. During the training phase, location fingerprints are collected in a regular spatial grid. During runtime, signal strength measurements received from the mobile device are accumulated and compared with the fingerprints obtained during training in order to perform localization.

Location estimation methods can be classified into three categories, namely, the deterministic, the probabilistic, and the recently introduced approaches based on spatial sparsity. In [5], we proposed a compressed sensing (CS) scheme that outperforms traditional localization techniques in terms of location accuracy. Although, fingerprint-based systems achieve the best performance, the time and effort required during the initial calibration and subsequent recalibrations remains their major disadvantage.

In our previous work [6], we proposed a reduced effort calibration phase for fingerprint-based systems. Particularly, we reduced the number of required RSS fingerprints by sensing a subset of the available channels in a WLAN. In this work, we extend our previous study by proposing a novel recalibration procedure that dynamically adapts to the envi- 
ronmental changes while minimizing the recalibration effort. Our key observation is that in non-stationary indoor environments, the training fingerprints are constantly changing over time while the subsequent measurements may explicitly depend on past observations. Thus, we exploit the spatiotemporal correlations of the training fingerprints to reduce the training phase effort by reconstructing the signature map from fewer RSS measurements.

The organization of the paper is as follows: In Section 2 we present a short overview of related work while in Section 3 we discuss the motivation of the proposed framework. Section 4 presents the necessary MC background and Section 5 describes the proposed recalibration technique based on Dynamic Matrix Completion. Experimental results are provided in Section 6, while we conclude in Section 7.

\section{RELATED WORK}

Fingerprint-based localization systems require significant effort, resources, and time in order to create the signature maps that represent the signal characteristics in the physical space. Achieving a satisfactory localization performance requires the recalibration of the signature map every time that environmental changes, which affect the power or the number of APs, occur in the area of interest. Recent efforts in the field have shown a growing interest in reducing the training phase without significantly compromising the quality of the location estimation.

Authors in [4] adopt traditional interpolation approaches to complete the training map using fingerprints received from a small number of training points. In order to recalibrate the localization system on-line, authors in [7] utilize the RSS measurements transmitted between anchor nodes. Then, a set of clusters is used and the signature map is updated via interpolation techniques. In [8], the number of labeled data is minimized by extracting information from a set of unlabeled data, and an interpolation approach is used in order to reinforce the signature map. Interpolation is based either on intuitive guidelines or on linear regression techniques.

To reduce the maintenance effort of the training phase, in [9] the signature map is estimated dynamically based on the runtime measurements. For this purpose, neighboring APs exchange RSS measurements and utilize Gaussian regression to model the path loss and the corresponding RSS values. Although [9] offers a recalibration phase, the system requires specific hardware. In contrast, our approach does not require any extra hardware.

Recent works try to decrease the training effort by crowdsourcing. Zee in [10] leverages the inertial sensor of a mobile phone to get a rough movement pattern by step counting and compares these measurements to prior information about the environment. On the contrary, our proposed system exploits the already existing WiFi infrastructure and most importantly it provides a dynamic recalibration of the indoor environment.

\section{MOTIVATION}

One of the major shortcomings of fingerprint-based systems is the exhaustive survey and maintenance of the signature map, a task that requires substantial cost and labor. To address this problem, we use a grid representation of the space and we propose to perform random sampling, where the mobile device (MD) collects RSS measurements from pseudo-randomly selected cells in the area of interest. Sub-sampling in the space domain reduces the calibration effort and consequently energy consumption at the MD. While random sensing has numerous benefits, recovering unobserved measurements is feasible if spatio-temporal correlations between the RSS fingerprints exist.

In indoor environments, signal strength measurements are affected by path loss and shadowing effects, which represent the signal degradation, due to the distance traveled and the obstacles, respectively. Thus, a key observation is that training fingerprints are spatially correlated in the sense that training locations in proximity have similar feature vectors. To support this claim, we created RSS fingerprints from 15 APs at 144 different positions. For this purpose we adopt the multiwall path loss model for signal propagation in [11]. Observe in Figure 2 that only a small number of the singular values are actually dominant, indicating the low rank property of the signature map.

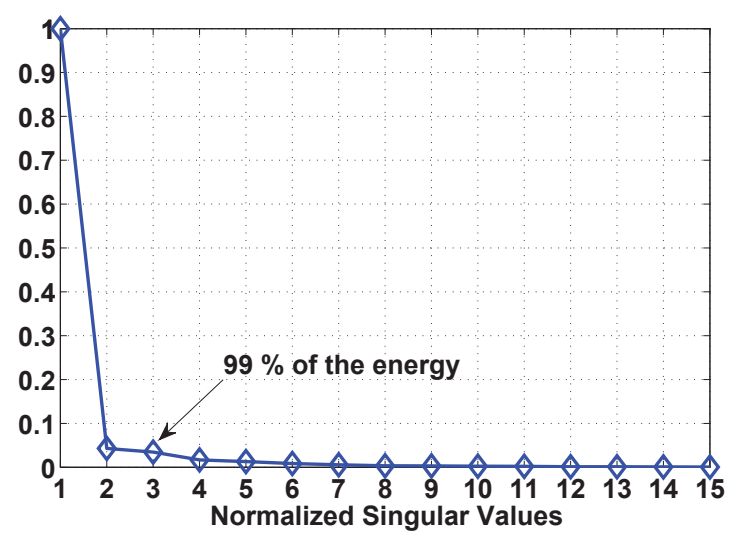

Fig. 1. Singular values of the signature map at a random time instance. The rank of the signature map equals the number of non-zero singular values.

Additionally, in dynamic indoor environments the signal strength fingerprints change over time, even at fixed positions, due to multipath phenomena. Signal fluctuations resulting from environmental dynamics, such as the presence of obstacles, walls, and doors, remains the main challenge for fingerprint based systems. The degree of correlation between RSS measurements over different time instances varies according to the temporal variation characteristics of the dynamic phenomena. Particularly, a strong temporal correlation is ob- 
served with the presence of the LOS path but it is degraded when LOS is obstructed. Compared to the state-of-the-art recalibration techniques, our work exploits the spatio-temporal correlations of the RSS fingerprints in order to recover the unobserved measurements.

\section{MATRIX COMPLETION}

Matrix Completion (MC) builds on the observation that a matrix which is low rank or approximately low rank can be recovered using just a subset of randomly observed data [12]. Denote the matrix $M \in \mathbb{R}^{J \times D}$ that we would like to recover as precisely as possible. The goal according to the MC formulation is to recover $M$ using the available measurements that can be gathered by the linear operator $\mathcal{A}: \mathbb{R}^{J \times D} \rightarrow \mathbb{R}^{N}$ such that:

$$
\boldsymbol{y}=\mathcal{A}(\boldsymbol{M}),
$$

where $\boldsymbol{y} \in \mathbb{R}^{N}$.

Clearly, the recovery of the $J \times D$ entries of an arbitrary matrix $M$ is impossible from a number of measurements $N$, when $N \ll J \times D$. This occurs as the linear system of equations described in (1) is underdetermined. Fortunately, the MC framework shows that such a recovery is possible, in the case where the rank of matrix $M$ is small enough compared to its dimensions. We recall that the rank of a matrix is defined as the number of its linearly independent rows or columns.

In order to recover the unknown low-rank matrix $M$, the following optimization problem needs to be solved

$$
\min \{\operatorname{rank}(\hat{\boldsymbol{M}}) \quad: \quad \mathcal{A}(\hat{\boldsymbol{M}})=\mathcal{A}(\boldsymbol{M})\}
$$

The general rank minimization problem is NP-hard. However, it can be replaced by the tightest nuclear norm minimization problem given that the linear map $\mathcal{A}$ satisfies a modified Restricted Isometry Property (RIP), i.e., uniform random sampling is performed in rows and columns. Consequently, finding the matrix with the lower-rank translates to the following optimization problem:

$$
\min \left\{\|\hat{M}\|_{\star}: \mathcal{A}(\hat{M})=\mathcal{A}(\boldsymbol{M})\right\},
$$

where the nuclear norm is defined as the sum of the singular values of $\boldsymbol{M}$, i.e. $\|\boldsymbol{M}\|_{\star}=\sum_{k=1}^{\min \{J, D\}} \sigma_{k}(\boldsymbol{M})$ and $\sigma_{k}(\boldsymbol{M})$ is the $k$ th largest singular value. Specifically, the recovery of the unknown matrix is feasible from $N \geq c D^{6 / 5} r \log (D)$ random measurements, where $D>J$ and $\operatorname{rank}(\boldsymbol{M})=r$.

The problem in (3) can be treated as a general convex optimization problem and solved by any off-the-shelf interior point solver (e.g., CVX [13]), after being reformulated as a semidefinite program. Additionally, efficient algorithms have been proposed for solving the low rank matrix completion problem for massive data sets, e.g., Singular Value Thresholding (SVT) [14].

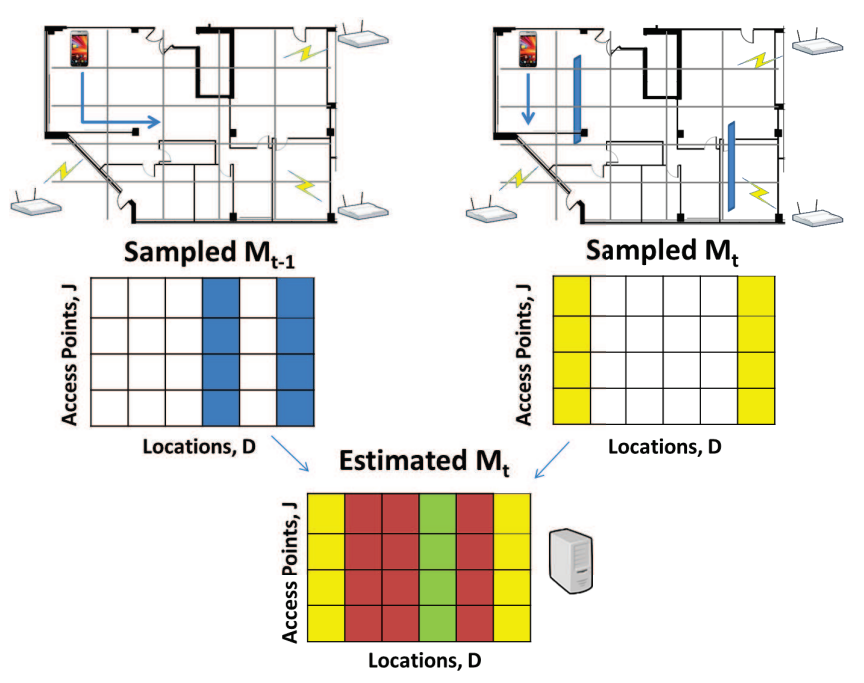

Fig. 2. A visual illustration of the proposed recalibration technique during the training phase. The MD randomly selects locations at time $t$, i.e. cells of grid here, to create RSS fingerprints from the packets transmitted from APs. The Location Server performs the Dynamic Matrix Completion to complete the missing fingerprints.

\section{PROPOSED DYNAMIC RECALIBRATION TECHNIQUE}

In this Section, we describe our proposed recalibration approach, which considers the spatio-temporal correlations of the RSS fingerprints among the APs. Consider a typical WLAN scenario with a MD equipped with an active wireless adapter card. The MD connected to a channel, periodically receives beacons sent by the APs at that channel and records its RSS value. Moreover, we consider a Location Server that is wireless connected with the MD.

Fingerprint-based techniques discretize the spatial space into a finite set of $D$ equivalent size cells, where each cell of the grid corresponds to a physical position. During recalibration at time $t$, the MD moves to the various cells and collects signal strength measurements from the $J$ APs that cover the area of interest. This procedure results in the generation of the signature map $\boldsymbol{M}_{t}$ represented as:

$$
\boldsymbol{M}_{t}=\left(\begin{array}{cccc}
P_{1,1, t} & P_{1,2, t} & \cdots & P_{1, D, t} \\
P_{2,1, t} & P_{2,2, t} & \cdots & P_{2, D, t} \\
\vdots & \vdots & \ddots & \vdots \\
P_{J, 1, t} & P_{J, 2, t} & \cdots & P_{J, D, t}
\end{array}\right)_{J \times D}
$$

where $P_{j, i, t}$ corresponds to the mean value of the RSS measurements received from the $j$ th AP at location $i$ at time $t$.

In dynamic indoor environments, the RSS fingerprints suffer from large variations over time, even in fixed positions, caused by multipath phenomena. The relationship of RSS measurements between path-loss and distance over time can be expressed as:

$$
\boldsymbol{y}_{t}=\boldsymbol{C} \boldsymbol{y}_{t-1}+\boldsymbol{\epsilon}
$$


where $\boldsymbol{y}_{t}, \boldsymbol{y}_{t-1} \in \mathbb{R}^{J \times 1}$ represent the measured RSS values at time $t$ and $t-1$, respectively, received at a fixed position [7]. $C \in \mathbb{R}^{J \times J}$ expresses the temporal correlation of the pathloss for specific distances, while $\epsilon$ indicates the environmental noise.

Efficient calibration of the signature map translates to periodically sensing the area of interest in order to capture the dynamic indoor propagation phenomena. The proposed Dynamic Matrix Completion framework is able to recover the unknown matrix at time $t$ under the assumption that the matrix is low-rank and follows a random sampling process. During training, the MD instead of visiting the whole workspace at time $t$, randomly, with equal probability, selects $K$ cells, where $K<D$. Once the selection is performed, the MD creates training fingerprints only for a subset of the area. It is obvious that spatial sub-sampling will result in an overall reduction of the calibration time.

Minimizing the number of visiting cells results in the incomplete signature map $M_{t}$. Particularly, the MD receives a subset $\Omega_{t} \subseteq[J] \times[D]$ of $M_{t}$ 's entries, where $|\Omega|_{t}=K \times J$. Let $\mathcal{A}_{t}$ be the sampling operator defined by

$$
\left[\mathcal{A}_{t}(\boldsymbol{M})\right]_{j, i}=\left\{\begin{aligned}
P_{j, i}, & (j, i) \in \Omega_{t} \\
0, & \text { otherwise. }
\end{aligned}\right.
$$

$\Omega_{t}$ is a subset of the complete set of entries $[J] \times[D]$. Moreover, $\Omega_{t} \cup \Omega_{t}^{C}=[J] \times[D]$. The complementary sampling operator $A_{t}^{C}\left(M_{t}\right)$ collects the unobserved measurements at time $t$. Additionally, we define the sampling operator $A^{I}=$ $A_{t-1} \cap A_{t}^{C}$ as the intersection of the training measurements of the cells visited at time $t-1$ and not at time $t$.

Effective location estimation requires the recovery of the signature map (4) that will be used during the runtime phase. In order to recover the signature map, we exploit the RSS samples received on previous time instances. Consequently, the Localization Server recovers the signature map at time $t$ based on the current partial measurements while also incorporating the information of the measurements obtained in the past (i.e. at time $t-1$ ). The proposed Dynamic MC method searches for the matrix $\boldsymbol{M}_{t}$ that has the minimum nuclear norm, subject to the values of $M_{t} \in \Omega_{t}$ being equal to the observed measurements at time $t$, and the sampled values at time $t-1$ being correlated with values at time $t$ via the matrix $C$ (eq. 5).

To enforce this constraint, the original matrix $M_{t}$ and the matrix $\mathrm{C}$ can be recovered as the solution of the following optimization problem:

$$
\begin{aligned}
\min _{\hat{\boldsymbol{M}}_{t}, \boldsymbol{C}} & \left\|\hat{\boldsymbol{M}}_{t}\right\|_{\star} \\
\text { subject to } & \left\|\mathcal{A}_{t}\left(\hat{\boldsymbol{M}}_{t}\right)-\mathcal{A}_{t}\left(\boldsymbol{M}_{t}\right)\right\|_{F}^{2} \leq \epsilon_{1} \\
& \left\|\mathcal{A}^{I}\left(\boldsymbol{C} \cdot \boldsymbol{M}_{t-1}\right)-\mathcal{A}^{I}\left(\boldsymbol{M}_{t}\right)\right\|_{F}^{2} \leq \epsilon_{2},
\end{aligned}
$$

where $\hat{M}_{t}$ is the recovered signature map at time $t, \epsilon_{1}, \epsilon_{2} \geq 0$ represent the tolerance in approximation error and $\|\cdot\|_{F}$ de- notes the Frobenious norm. The matrix $C$ expresses the relationship between the values of $M_{t} \in \Omega_{t}^{C} \cap \Omega_{t-1}$. Particularly, $C$ is changing as the number of the common training cells at time $t-1$ and $t$ increases. The more orthogonal the sampling operators are over time the more accurate the matrix $\boldsymbol{C}$ is.

The problem in (7) is a general convex optimization problem and can be solved by an off-the-shelf interior point solver, such as CVX.

\section{EXPERIMENTS}

The benefits and characteristics of the proposed recalibration technique based on Dynamic Matrix Completion are studied and analysed through simulations. Two different performance metrics are considered, namely, the reconstruction quality of the recovered signature map with respect to the original one and the corresponding localization error. The effectiveness of the proposed scheme is investigated in various environments and network characteristics. We compare the proposed technique with the traditional MC approach that serves as a baseline ${ }^{1}$. Regarding the reconstruction quality, the error was measured as $\left\|\hat{M}_{t}-M_{t}\right\|_{F} /\left\|M_{t}\right\|_{F}$. To evaluate the impact of the proposed technique in location estimation, the Nearest Neighbour in Signal Space (NNSS) algorithm was employed $[3,4]$.

According to the multi-wall path loss model [11], the received power at the $i$ th position from the $j$ th AP is given by

$$
P_{r}=P t-L_{0}-10 n \log \left(d_{i j}\right)-\sum_{w=1}^{W} k_{w} \cdot L_{w}-s_{i}
$$

where $L_{0}$ is the path loss at distance $1 \mathrm{~m}, n$ denotes the path loss exponent, $d_{i j}$ is the Euclidean distance between the $j$ th and the $i$ th cell. $k_{w}$ and $L_{w}$ are the number and loss coefficients due to the obstacles, $W$ denotes the number of obstacles while $s_{i}$ describes the shadowing effect. We chose $L_{0}=37.5 \mathrm{dBm}, n=3$ for NLOS, and $L_{w}=3.4 \mathrm{~dB}$ for light walls/obstacles. The shadowing variable is a zero-mean Gaussian random variable (in $\mathrm{dB}$ ) with standard deviation $\sigma=2 d B$. $P_{t}$ is the AP transmit power, which is fixed at $15 \mathrm{dBm}$ for IEEE $802.11 \mathrm{~b}$ based WLANs. The values of $\epsilon_{1}$ and $\epsilon_{2}$ are equal to $1 e-03$ and $1 e-01$, respectively. We considered an $144 \mathrm{~m}^{2}$-wide area and a $1 \mathrm{~m} \times 1 \mathrm{~m}$ grid-spacing ( $D=144)$, covered by 15 APs $(J=15)$. Each data point in the results is averaged over 20 independent trials.

For the next experiment, we create changing conditions over time by introducing one obstacle in the middle of the room. Figures 3-4 indicate the recovery of the signature map based on (7) and the corresponding location error as a function of the number of visiting cells. We observe that the performance of the proposed dynamic recalibration scheme is improved both in reconstruction and location error, as the

\footnotetext{
${ }^{1}$ Detail performance analysis concerning the effectiveness of the MC calibration technique vs. the Interpolation method can be found in [6].
} 


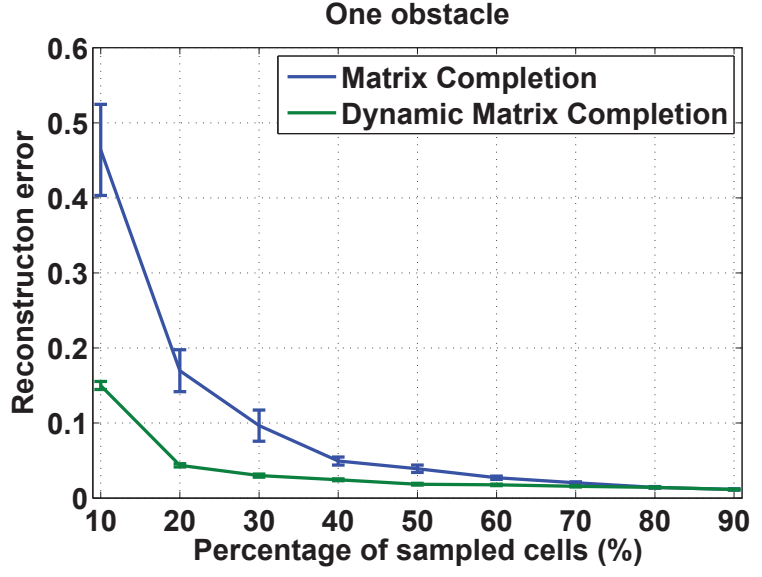

Fig. 3. The reconstruction error of the signature map as a function of the number of visiting cells. The experiment involves 15 available APs. Dynamic MC achieves the lowest reconstruction error especially for a small number of visiting cells.

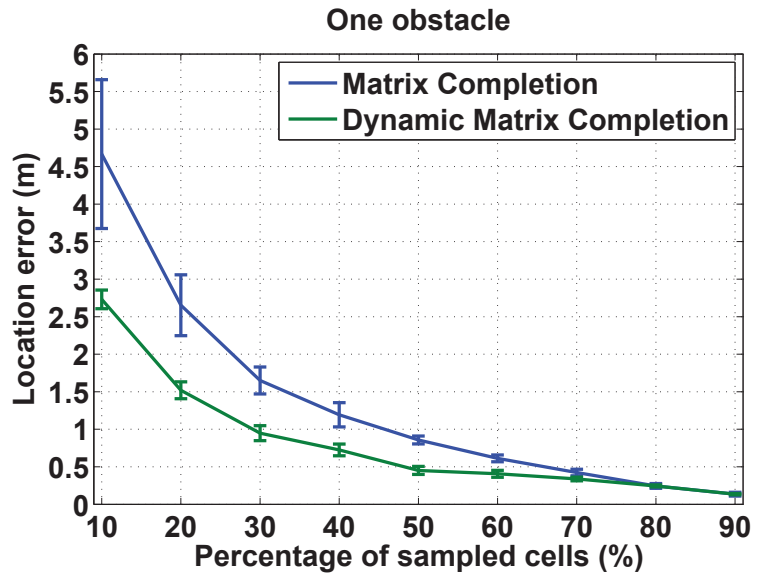

Fig. 4. The location error, during runtime, for 15 APs as a function of the number of visiting cells. Observe that the proposed recalibration technique results in lower location errors.

number of visiting cells increases. Observe that the dynamic approach is able to achieve lower reconstruction and location error as compared to the MC approach, especially for small sampling ratios. Particularly, for the NSSS, dynamic MC leads to improvements of $42 \%$ (i.e., $2 \mathrm{~m}$ ) over the location error achieved by the MC approach for sampling $10 \%$ of the total number of cells.

Figures 5-6 illustrate the performance of the proposed method as a function of the total number of available APs, when the sampling ratio of the received RSS fingerprints is 0.3. The total number of APs that cover the workplace affects the structure of the signature map. Specifically, as the number of APs increases, the accuracy of the proposed technique increases. Indeed, as the number of available APs increases, more correlated RSS fingerprints are produced resulting in

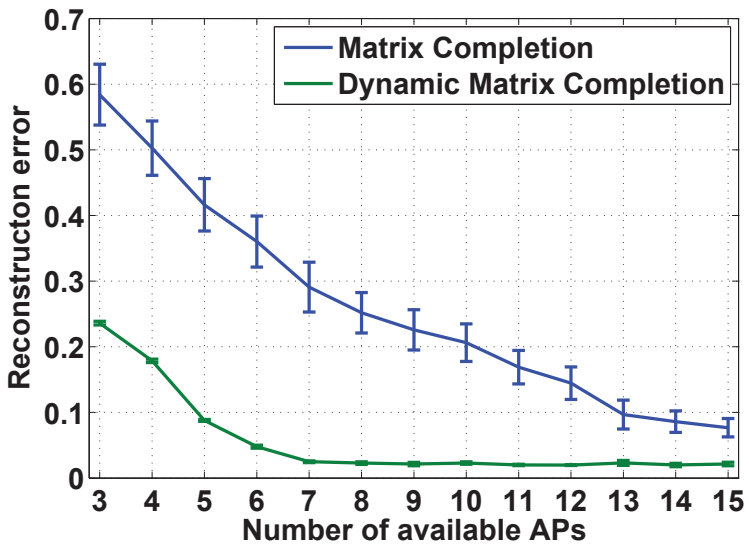

Fig. 5. Signature map reconstruction, during training phase, as a function of the total number of available APs covering the area of interest. In this experiment, we visit $30 \%$ of the cells in the testbed area. The number of APs defines the structure of the signature map.

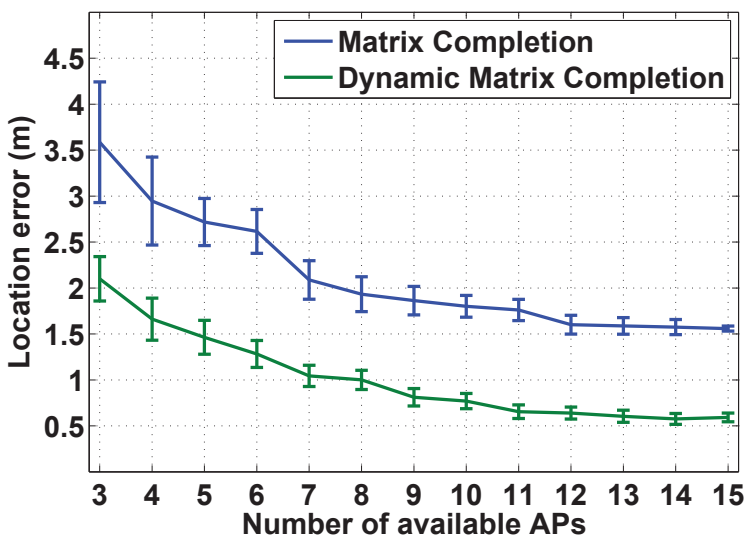

Fig. 6. Location error, during runtime phase, as a function of the total number of available APs. In this experiment, we visit $30 \%$ of the cells in the testbed area.

a signature map that has an even lower rank relative to its dimensions. Interestingly, the localization performance is not highly affected after a certain number of APs. Observe that the dynamic MC framework converges faster to the optimum number of APs.

Finally, we investigated the robustness of the proposed recalibration technique under different environmental conditions. For this purpose, we introduced five obstacles in the workspace at time $t$. Figures 7-8 demonstrate the reconstruction and the location error, respectively. In this setup, we considered 15 available APs. It is clear that, for complicated scenarios, the dynamic approach results in lower reconstruction and location errors, especially for small sampling ratios. The dynamic MC is affected by the increased noise. Particularly, we observe that after a certain sampling ratio, the prior information slightly increases the reconstruction accuracy. This 


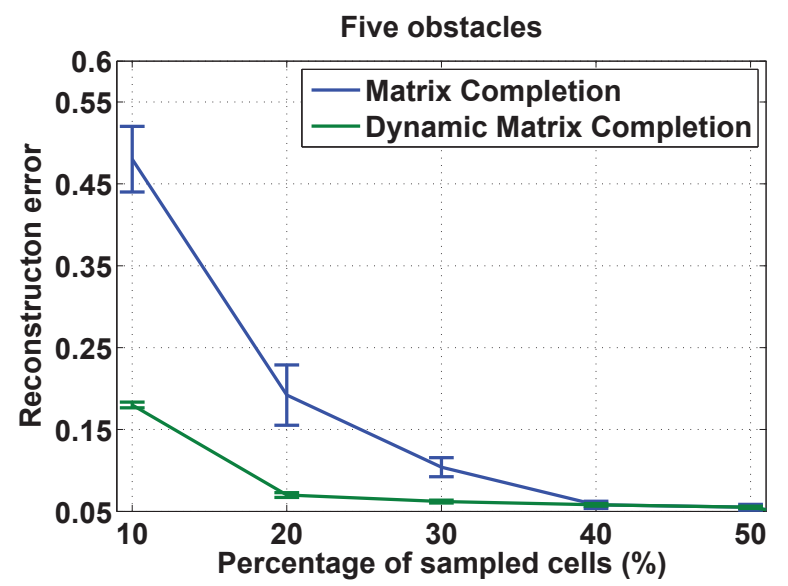

Fig. 7. Reconstruction error as a function of the number of visiting cells in the extreme case scenario of introducing five obstacles at time $t$. The experiment involves 15 APs. The proposed Dynamic MC results in lower reconstruction errors even in complicated scenarios.

behavior is expected in adaptive systems, where previous information becomes less reliable as the noise increases.

\section{CONCLUSIONS}

In this paper, we presented a novel recalibration procedure for RSS fingerprint-based localization systems. The proposed technique, based on space sub-sampling, exploits the inherent spatial correlation structure among the RSS fingerprints, while considering prior information from RSS measurements collected in the past. We relaxed the acquisition and communication requirements while the time required for recalibration was significantly reduced. We adopted a Dynamic Matrix Completion approach to recover the original signature map just from visiting a subset of cells in the area of interest. The experimental results indicated that our Dynamic MC reconstruction method can achieve superior performance when compared to a straight MC technique, in terms of reconstruction error and localization accuracy. Future work will investigate theoretical bounds that quantify the impact of prior information on the reconstruction quality.

\section{REFERENCES}

[1] H. Liu et al., "Survey of wireless indoor positioning techniques and systems," IEEE Trans. on Systems, Man, and Cybernetics, vol. 37, no. 6, pp. 1067-1080, 2007.

[2] J. Yang and Y. Chen, "Indoor localization using improved rss-based lateration methods," in Proc. IEEE Global Telecommun. Conf., Honolulu, Hawaii 2009, pp. $1-6$.

[3] P. Bahl and V. N. Padmanabhan, "Radar: an in-building rf-based user location and tracking system," in Proc. IEEE 19th Ann. Conf. Comput. Commun. Soc., Tel Aviv, 2000, pp. 775-784.

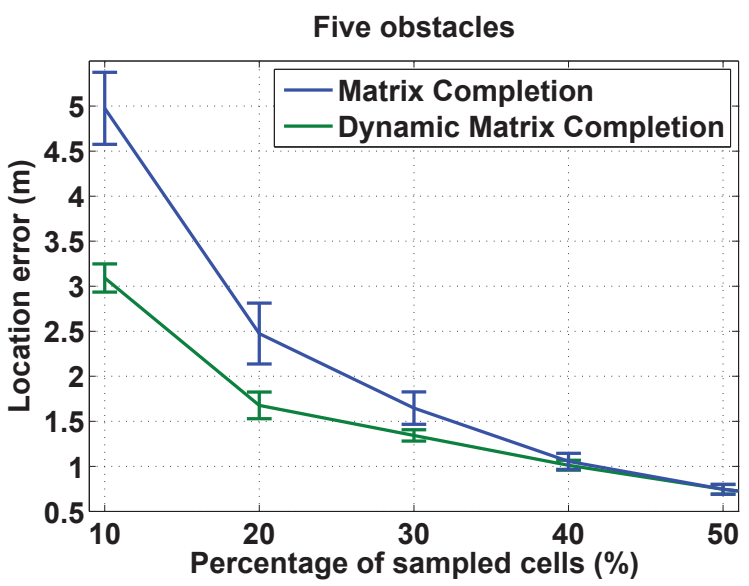

Fig. 8. Location error as a function of the number of visiting cells in the extreme case scenario of introducing five obstacles at time $t$. The experiment involves 15 APs.

[4] B. Li, J. Salter, A. G. Dempster, and C. Rizos, "Indoor positioning techniques based on wireless lan," in Proc. AusWireless, 2006, pp. 13-16.

[5] S. Nikitaki and P. Tsakalides, "Localization in wireless networks based on jointly compressed sensing," in Proc. 20th IEEE European Signal Process. Conf., Barcelona, 2011.

[6] S. Nikitaki, G. Tsagkatakis, and P. Tsakalides, "Efficient training for fingerprint based positioning using matrix completion," in Proc. 20th IEEE European Signal Process. Conf., Boucharest, 2012, pp. 195-199.

[7] C-C Lo, L-Y Hsu, and Y-C Tseng, "Adaptive radio maps for pattern-matching localization via inter-beacon co-calibration," Journal on Pervasive Mob. Comput., vol. 8, no. 2, pp. 282-291, 2012.

[8] X. Chai and Q. Yang, "Reducing the calibration effort for probabilistic indoor location estimation," IEEE Trans. Mobile Comput., vol. 6, no. 6, pp. 649-662, 2007.

[9] M. Atia, A. Noureldin, and M. Korenberg, "Dynamic online-calibrated radio maps for indoor positioning in wireless local area networks," IEEE Trans. Mobile Comput., vol. 99, no. PrePrints, 2012.

[10] A. Rai, K.K. Chintalapudi, V.N. Padmanabhan, and R. Sen, "Zee: Zero-effort crowdsourcing for indoor localization," in Proc. of the 18th Ann. Int. Conf. on Mobile Comput. and Netw., New York, NY, 2012, pp. 293-304.

[11] M. Lott and I. Forkel, "A multi-wall-and-floor model for indoor radio propagation," in Proc. IEEE 54th Vehicular Techn. Conf., Atlantic City, 2001, pp. 464-468.

[12] E. Candès and B. Recht, "Exact matrix completion via convex optimization," Foundations of Compuational Mathematics, vol. 9, pp. 717-772, 2009.

[13] M. Grant and S. Boyd, "CVX: Matlab software for disciplined convex programming, version 1.21," 2011.

[14] J. Cai, E. Candès, and Z. Shen, "A singular value thresholding algorithm for matrix completion," SIAM J. on Optimization, vol. 20, pp. 1956-1982, 2010. 